\title{
Correction to: Radiopharmaceutical tracers for cardiac imaging
}

\author{
Osamu Manabe, MD, PhD, ${ }^{a}$ Tatsuya Kikuchi, PhD, ${ }^{b}$ Arthur J. H. A. Scholte, \\ MD, PhD, ${ }^{c}$ Mohammed El Mahdiui, MD, ${ }^{c}$ Ryuichi Nishii, MD, PhD, ${ }^{d}$ \\ Ming-Rong Zhang, PhD, ${ }^{\mathrm{b}}$ Eriko Suzuki, LT, a and Keiichiro Yoshinaga, \\ $M D, P h D, F A C C$, FASNC ${ }^{d}$ \\ a Department of Nuclear Medicine, Hokkaido University Graduate School of Medicine, Sapporo, \\ Japan \\ b Department of Radiopharmaceutical Development, National Institutes for Quantum and \\ Radiological Science and Technology, National Institute of Radiological Sciences, Chiba, Japan \\ c Department of Cardiology, Leiden University Medical Center, Leiden, The Netherlands \\ d Diagnostic and Therapeutic Nuclear Medicine, National Institutes for Quantum and Radiolog- \\ ical Science and Technology, National Institute of Radiological Sciences, Chiba, Japan
}

doi: $10.1007 / \mathrm{s} 12350-018-1184-0$

\section{CORRECTION TO: J NUCL CARDIOL}

HTTPS://DOI.ORG/10.1007/S12350-017-1131-5

Regrettably the original version of the above article contained errors in the three chemical structures presented in the 'Atherosclerosis imaging' section of
Table 5, namely: ${ }^{99 \mathrm{~m}} \mathrm{Tc}$ annexin $\mathrm{V},{ }^{68} \mathrm{Ga}$ DOTATATE, and ${ }^{64} \mathrm{Cu}$ DOTATATE; the chemical structures have been corrected in Table 5 presented here. In addition, the radiopharmaceutical for isotope ${ }^{67} \mathrm{Ga}$ has been corrected to ${ }^{67} \mathrm{Ga}$ citrate, and many of the radiopharmaceuticals presented at the end of the table have been corrected.

The original article can be found online at https://doi.org/10. 1007/s12350-017-1131-5.

Reprint requests: Keiichiro Yoshinaga, MD, PhD, FACC, FASNC, Diagnostic and Therapeutic Nuclear Medicine, National Institutes for Quantum and Radiological Science and Technology, National Institute of Radiological Sciences, 4-9-1 Anagawa, Inage-Ku, Chiba 263-8555, Japan; yoshinaga.keiichiro@qst.go.jp

J Nucl Cardiol 2018;25:1237-41.

$1071-3581 / \$ 34.00$

Copyright (c) 2018 American Society of Nuclear Cardiology. 


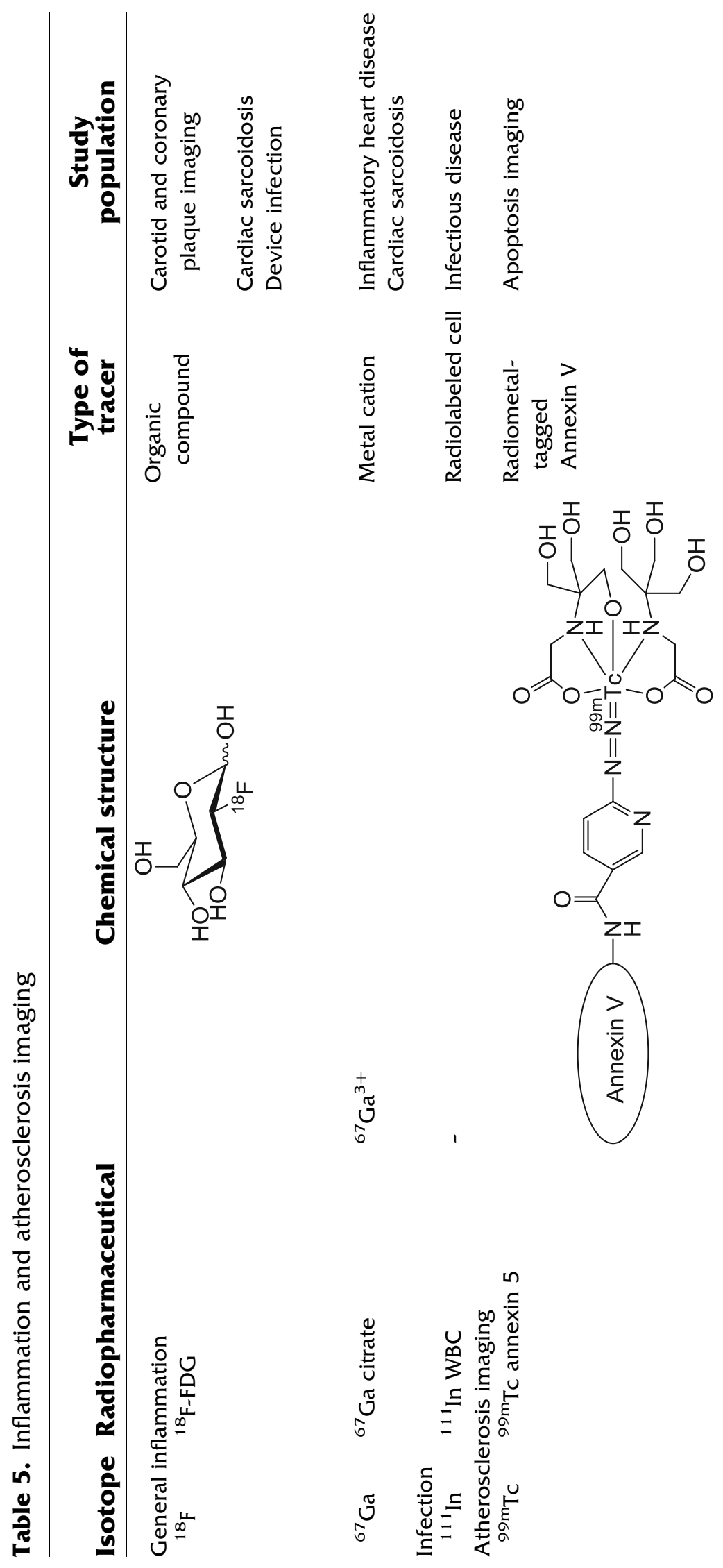



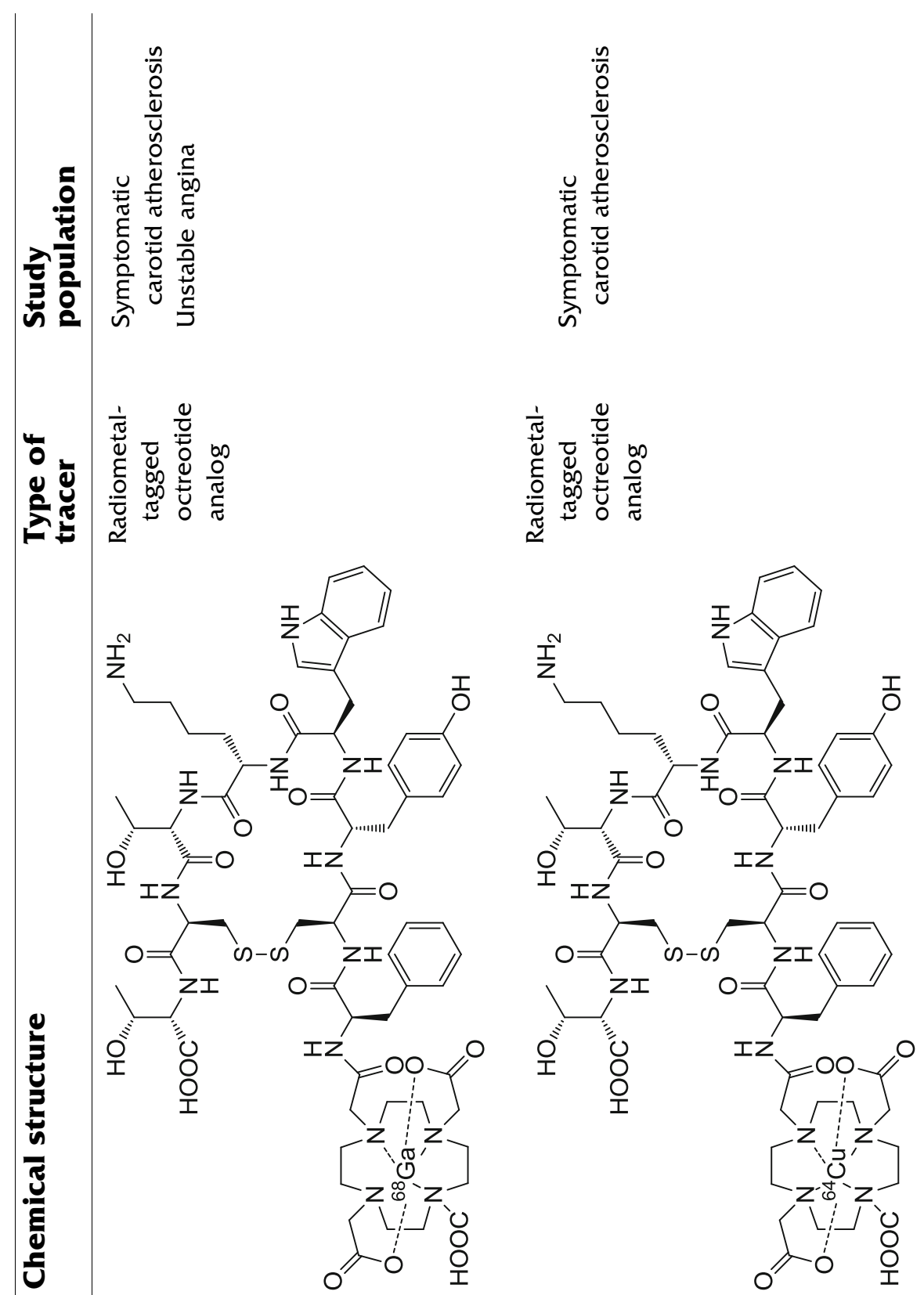

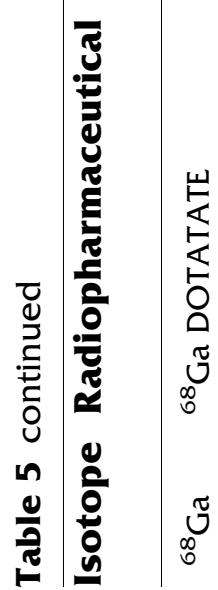

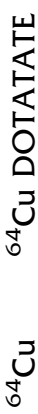



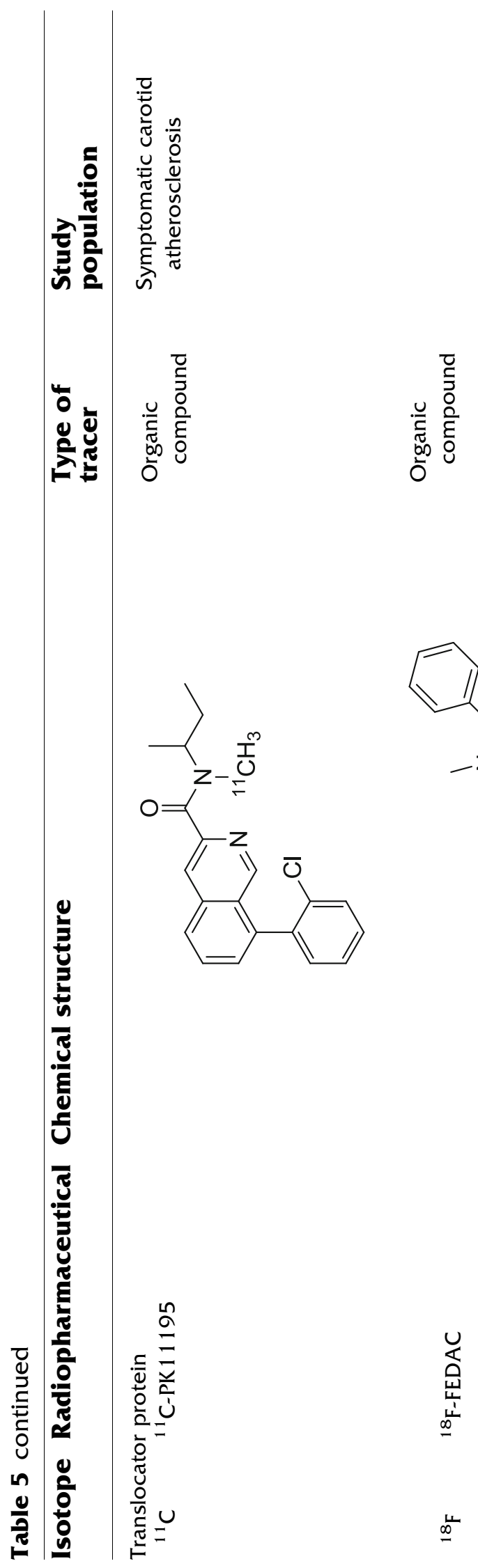

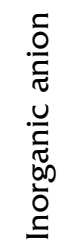
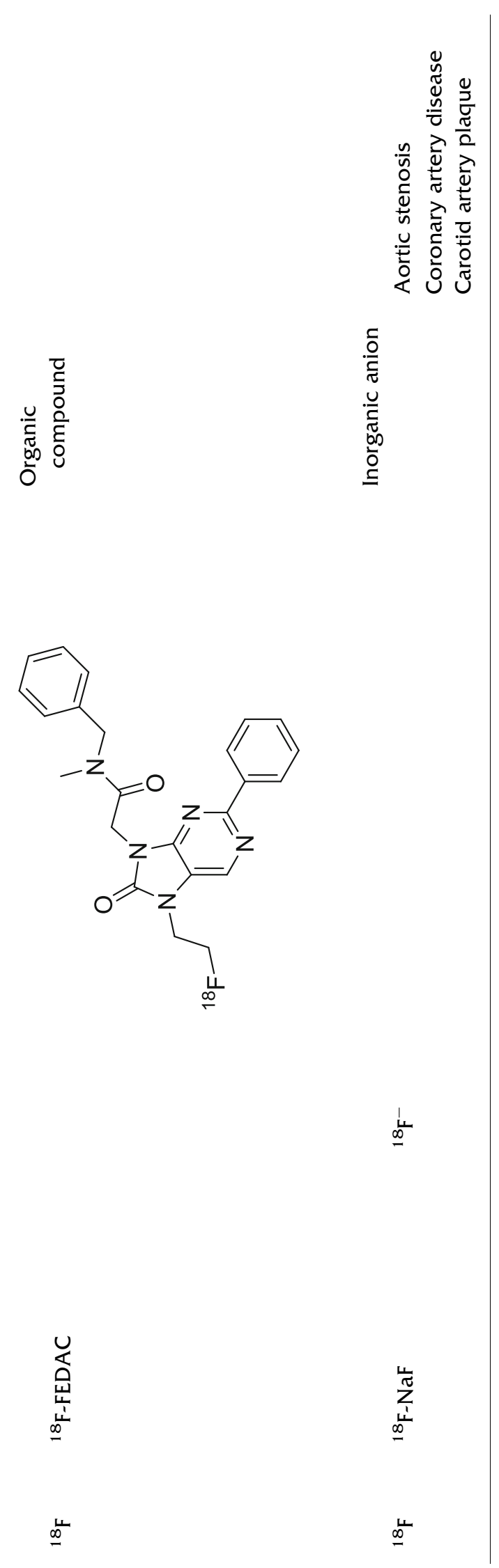


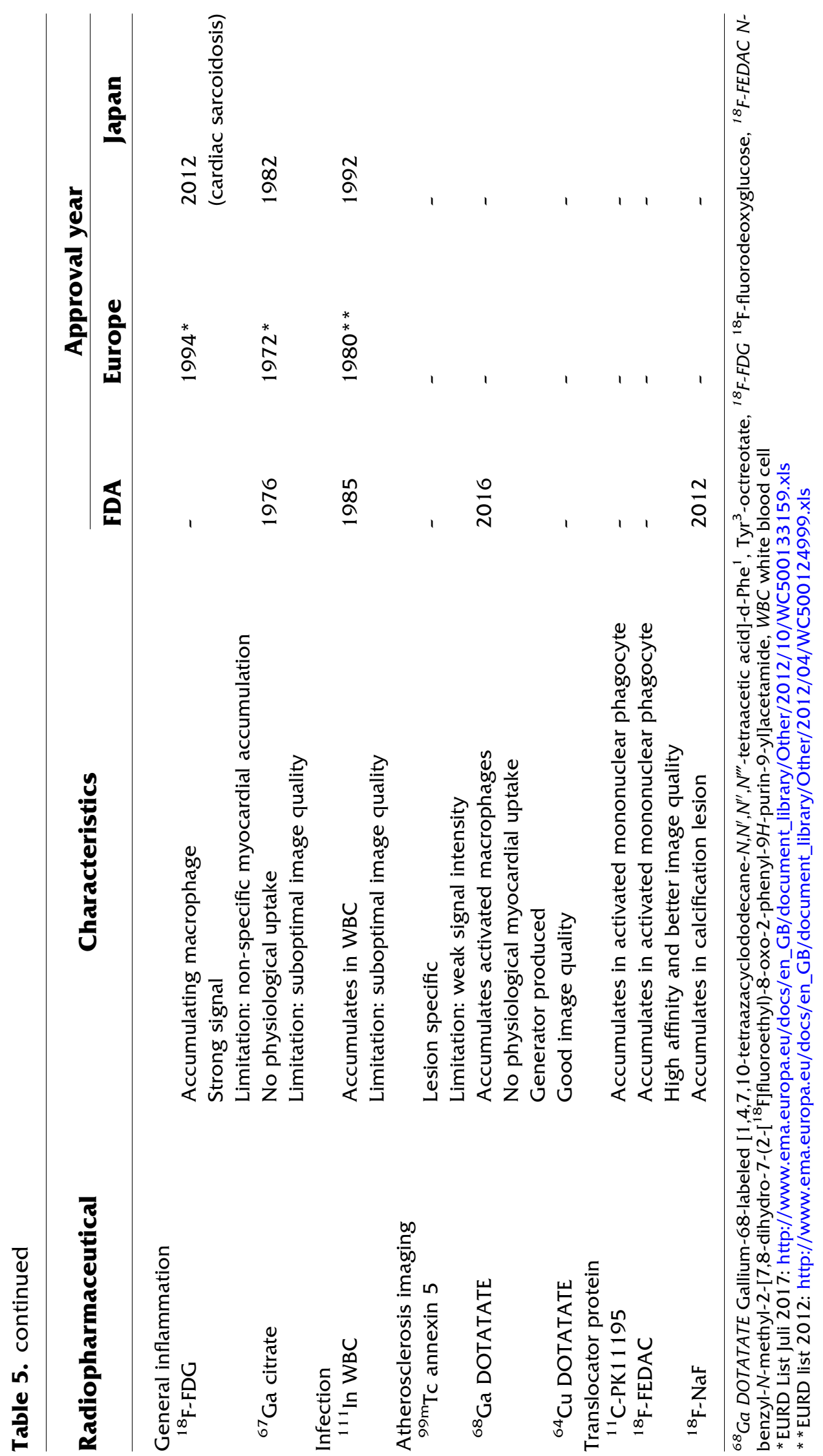

